\title{
Aerodynamic characterization of bio inspired corrugated wings
}

\begin{abstract}
Wings of a dragon fly found in nature possess corrugations on its surface and known to exhibit efficient aerodynamic characteristics. In the present work, aerodynamic analysis of bio inspired profiles corrugated A, corrugated B and NACA 0010 were studied using 2-D panel method and wind tunnel experimentation. Coefficient of lift, drag and glide ratio for all profiles were evaluated at two Reynolds numbers $1.9 \times 10^{4}$ and $1.5 \times 10^{5}$ and for angles of attack between $0^{0}$ to $16^{0}$ The panel method results have been validated using current and existing experiment data as well as with computational work from cited literature. A good agreement between experimental and panel methods were found for low angles of attack. The results demonstrated that till $8^{0}$ angle of attack higher lift coefficient and lower drag coefficient are obtainable for corrugated profiles compared to NACA 0010. Validation of surface pressure coefficients for all three profiles using panel method at $4^{0}$ angles of attack was done. Contours of non-dimensional pressure, velocity are demonstrated from $-10^{\circ}$ to $20^{0}$ angles of attack. For low Reynolds number flows good correlation between experiment data and computational methods showed that corrugated profiles exhibit better aerodynamic performance than NACA 0010.
\end{abstract}

Keywords: corrugated airfoil, lift coefficient, drag coefficient, panel method, reynolds number

\section{Introduction}

The nature gives effective, robust and straightforward solution for engineering problems and this practice is becoming very popular day by day. ${ }^{1}$ The deep understanding of nature, how it overcomes the problem of flight in natural flyers like birds and insects have led to tremendous improvements in the aerodynamics of manmade aerial vehicles. Creatures of flight such as birds and insects have inspired human to design aircrafts of various types and sizes. Specifically, the smooth airfoil shapes of familiar aircrafts are inspired by the bird's wing. ${ }^{2}$ The smaller aircrafts like unmanned aerial vehicles (UAVs) operate at significantly lower Reynolds number, where the performance of the conventional airfoils is no longer better. ${ }^{3}$ The aerodynamic effects of low Reynolds numbers on corrugated wings were studied by several researchers ${ }^{4-8}$ It was found that most insects' wings specifically dragonflies and locusts have high agility and maneuverability. These insects are capable to hover, climb, dive, land and take-off at different wind speeds and ambient conditions. Another investigation ${ }^{9}$ reported that the remarkable aerodynamic performance of dragonfly wings was caused by the muscular morphing and wing geometry specially the wing corrugation. Extensive biological and aerodynamic study has been carried out by many researchers to demonstrate the reasons for the superior flight by flapping motion of the wings and the corrugated wing profile (airfoil ${ }^{10}$ revealed that the flapping produces significant lift by generating low pressure leading edge vortex (LEV) while corrugated cross section, reduces the overall flow separations in both flapping and static motion. The effects of corrugations were demonstrated, and the results are reported in several experimental studies such as. ${ }^{11,12}$ These reports confirmed that the corrugation initiates an early transition to turbulent flow thus reducing separation and delay in stall. A better aerodynamic characteristic obtained using pleated airfoil at first sight seems to contradict with the idea of streamlined profiles used in conventional aircrafts. However recent studies by ${ }^{13-16}$, suggested otherwise. There also have been several studies related to aerodynamic forces which were conducted on actual dragonflies and dragonfly modeled airfoils to measure the
Volume 3 Issue I - 2019

\author{
DwivediYD,' Vasishta Bhargava² \\ 'Institute of Aeronautical Engineering, India \\ ${ }^{2}$ Sreyas Institute of Engineering \& Technology, India
}

Correspondence: Dwivedi YD, Institute of Aeronautical Engineering, Dundigal, Hyderabad, India, 50004, Email yddwivedi@gmail.com

Received: December 10, 2018 | Published: January 23, 2019 forces associated with both flapping and gliding modes of operation. Some of the more notable and recent studies for gliding flight of a dragonfly wing are the numerical and experimental studies conducted by ${ }^{17-22}$ More recently ${ }^{23-25}$ have performed 3-D computational study on different types of corrugated wings by using commercially available computational software. However, these studies have been primarily conducted at chord Reynolds number $10^{4}$ or less, which is considerably lower than the range used for MAVs. The aim of the present work is analyze two different types of corrugation in relation to coefficient of lift, drag and glide ratio, which are also relevant for micro aerial vehicles. From author's knowledge no studies have been conducted before on bio-inspired corrugated wing using panel method. The other aim is to explore whether the 2-D panel method can predict low Reynolds number flows fairly accurately and agree with experiment data conducted on similar profiles.

\section{Methodology \\ Numerical formulation}

Panel methods are designed for potential flows which are commonly used in analysis of 2D lifting flows that have negligible or low viscosity. For computational analysis, it is known that velocity potential function can be readily used to describe the aerodynamic flow field around the arbitrary shaped body than streamline function. This is because both for 2D and $3 \mathrm{D}$ analysis of flow, velocity field exists at every coordinate on surface, however for stream function the derivatives of velocity vector becomes complex for analysis of 3D flow. The basic approach involved in panel methods is a combination of source along with sink which have equal strength, $\mathrm{K}$ present in a uniform distributed flow pattern around an arbitrary body. Source strengths are defined in such a way that appears in the fluid field as streamlines at a uniform rate while sink disappear at a same rate ${ }^{26}$ For any given arbitrary shaped body, the geometry is divided into specific no of panels representing the surface. The panels represent the approximation of geometry surface, using straight line segments. For a given $\mathrm{AOA}, \alpha$, the steps involved are as follows 
Number the panels sequentially from $1 \ldots \mathrm{N}$

A. Calculate the center point also known as collocation or control point for defined panels representing the geometry.

B. Most appropriate boundary condition for airfoil profiles, using panel method involves impermeable flow perpendicular to the surface describing the profile

C. A set of unit vectors are applied in perpendicular and tangent directions,

D. Velocity field is derived by taking account of any two panels, $I$ $\& j$, source strength of individual panel $j$ induced on panel $I$ with respect to stream wise direction

E. After defining the local velocity vector for all the panels of the discretized airfoil surface, the influence coefficients are evaluated for each panel in perpendicular and tangential directions respectively.

F. Unknown source and vortex strengths are evaluated for each panel

G. Finally the tangential velocity vector field over the airfoil surface is calculated using the known values of influence coefficients
(Normal $\left(\mathrm{C}_{\mathrm{n}}\right)$ and tangential $\left(\mathrm{C}_{\mathrm{t}}\right)$ directions to chord line) matrix, source and vortex strength matrix vector. The tangential velocity vector is used to determine the surface pressure coefficient along the chord length of the airfoil surface.

It must be noted that in MATLAB for all the variables i.e. velocity and its components, matrices are essential and required to solve the system of discrete equations. The surface pressure coefficient can be expressed using the Eq. (1) Another boundary condition also known as "Kutta condition" is imposed to account for the flow near the trailing edge so that tangential velocity vector for trailing edge panels equal in magnitude on upper and lower surfaces of airfoil. The surfaces represented by the panels are typically solid rectangular or curvilinear areas upon which the above conditions are applied. Therefore the system of linear algebraic equations are solved for $\mathrm{N}$ unknown source strengths, $\sigma_{i}$, using the influence coefficients and written in matrix form as shown in flow chart for computational routine in Figure 1. The pressure acting at any collocation point $i$ on panel surface can be expressed in non-dimensional form as in Eq. (1)

$$
\mathrm{C}_{\mathrm{p}_{\mathrm{i}}}=1-\left[\frac{\mathrm{v}_{\mathrm{t}_{\mathrm{i}}},}{\mathrm{U}}\right]^{2}
$$

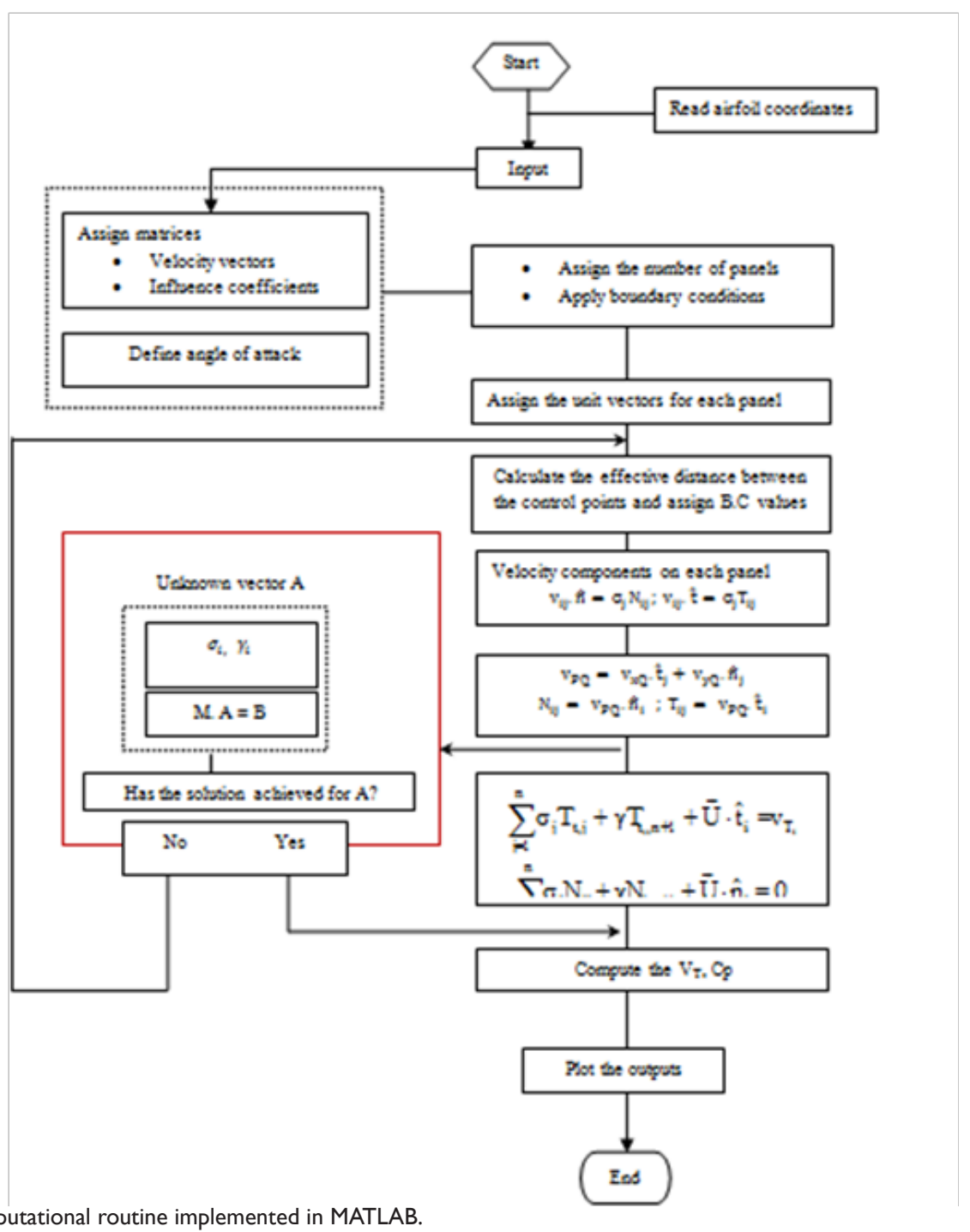

Figure I Flow chart of computational routine implemented in MATLAB. 
The number of panel nodes used in the simulation is 35 and the maximum panel angle is $76.77^{\circ}$ in airfoil geometry. The lift and drag coefficient for the standard airfoil specimens by Pamadi (2004) can be found using Eq. (2) \& Eq. (3)

$$
\begin{aligned}
& C_{L}=L\left(0.5 \rho U^{2} S\right) \\
& C_{D}=D\left(0.5 \rho U^{2} S\right)
\end{aligned}
$$

where $\rho$ is fluid density, $U$ is free stream velocity and $S$ is plan form area, the coefficients of total lift $C_{\mathrm{L}}$ and total drag $C_{\mathrm{D}}$. They are obtained using the pressure coefficient between upper and lower surfaces for each pane, multiplied with its panel length and integrating them at any given angle of attack. Further, the lift and drag coefficients could also be evaluated using normal and tangential force influence coefficients as given by Eq. (4) and Eq. (5)

$$
\begin{aligned}
& \mathrm{C}_{\mathrm{L}}=C_{n} \cdot \cos \alpha-C_{t} \cdot \sin \alpha \\
& \mathrm{C}_{\mathrm{D}}=C_{n} \cdot \sin \alpha+C_{t} \cdot \cos \alpha
\end{aligned}
$$

Aerodynamic performance of a wing during gliding can therefore be determined by Eq. (6)

$$
\epsilon_{R}=C_{L} / C_{D}
$$

\section{Geometry of profiles}

The geometries of corrugated profiles were selected based upon the previous experimental and numerical investigations ${ }^{27-29}$ It can be seen from Figure 2A, for corrugated A profile, the corrugations are observed throughout its chord length. For corrugated B profile, sharp peaks are present near the leading edge up to $30 \%$ of chord length followed by a broad hump present towards the trailing edge as shown in Figure 2B. The corrugated profile used by Kessel, Murphy and $\mathrm{Hu}$ resembles the shape of corrugated A profile used in the present study, at the leading edge, and up to $50 \%$ of chord length while the profile used by Ho and New (2016) resembles closely to the corrugated B profile. It is also identical to the profile 2 used by Kessel in his investigation. These similarities in profiles enable better validation of results with those obtained from the earlier computational investigations..$^{30}$

\section{Experimental setup}

The low speed subsonic open circuit wind tunnel facility was used for the present experimental work (Table 1). It has three main sections, an inlet section, nozzle and test section and a diffuser section at the exit of the tunnel. The dimensions of the sections and the corresponding principal functions are shown in Table 2. Schematic representation of the open circuit wind tunnel test section used for experiments is shown in Figure 3A. The smoke rake provided in contraction cone just before the test section is used for smoke flow visualization. Velocity and pressure distribution can be measured by the inclined tube manometer as shown in Figure 3B. These two limbs of the manometer are connected to the static pressure holes, one in the settling chamber just before contraction side and the other to the start of the test section. The reading in the manometer shows the dynamic head of the fluid in the test section and it serves as the reference for keeping the tunnel speed constant. The inclination of the manometer is kept at $30^{\circ}$ to the horizontal, at this angle the liquid column length change is twice to the vertical head, to provide better accuracy. Tunnel is also provided with Pitot-static tube, which can be traversed across the tunnel section. The fan is connected with variable speed motor, which is varied by AC controller and motor required 3 phase A.C power. Which is equipped with six component force balance, pressure distribution system, and manometer. The snapshot of full experimental setup is shown in Figure 4A and the fabricated corrugated A profile in wind tunnel is shown in Figure 4B.

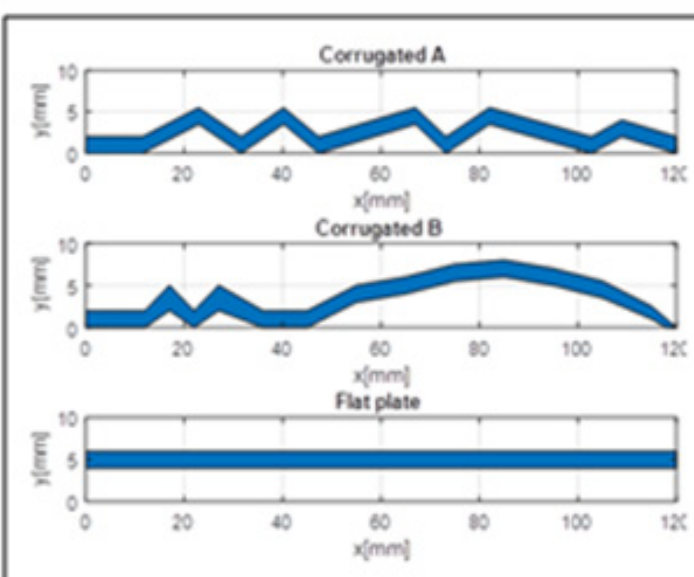

(a)

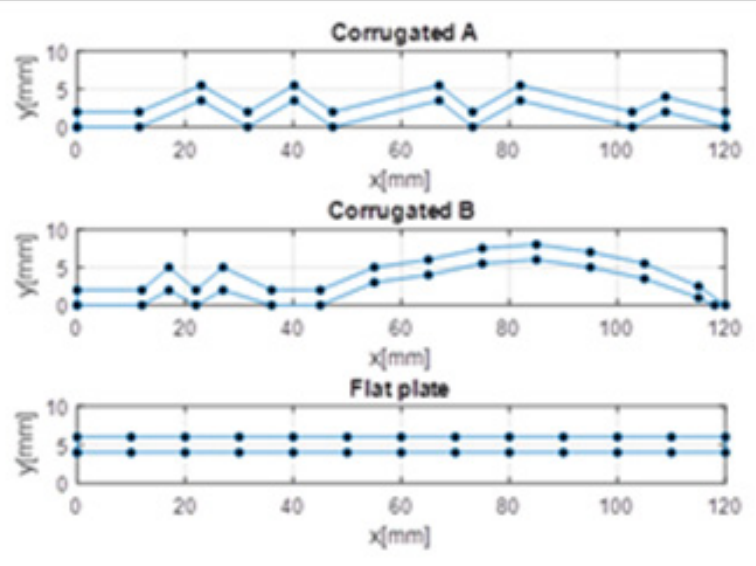

(b)

Figure 2 (A) Geometry of Corrugated A, Corrugated B, Flat plate, (B) panel approximation.

Table I Dimensions for NACA 0010 , Corrugated A and Corrugated B airfoil

\begin{tabular}{llll}
\hline Profile & Chord $[\mathbf{m}]$ & Max. thickness $[\mathbf{m}]$ & Thickness $/$ chord ratio [\%] \\
\hline NACA 0010 & 0.12 & 0.012 & 10 \\
Corrugated A & 0.12 & 0.006 & 5.0 \\
Corrugated B & 0.12 & 0.008 & 6.6 \\
\hline
\end{tabular}




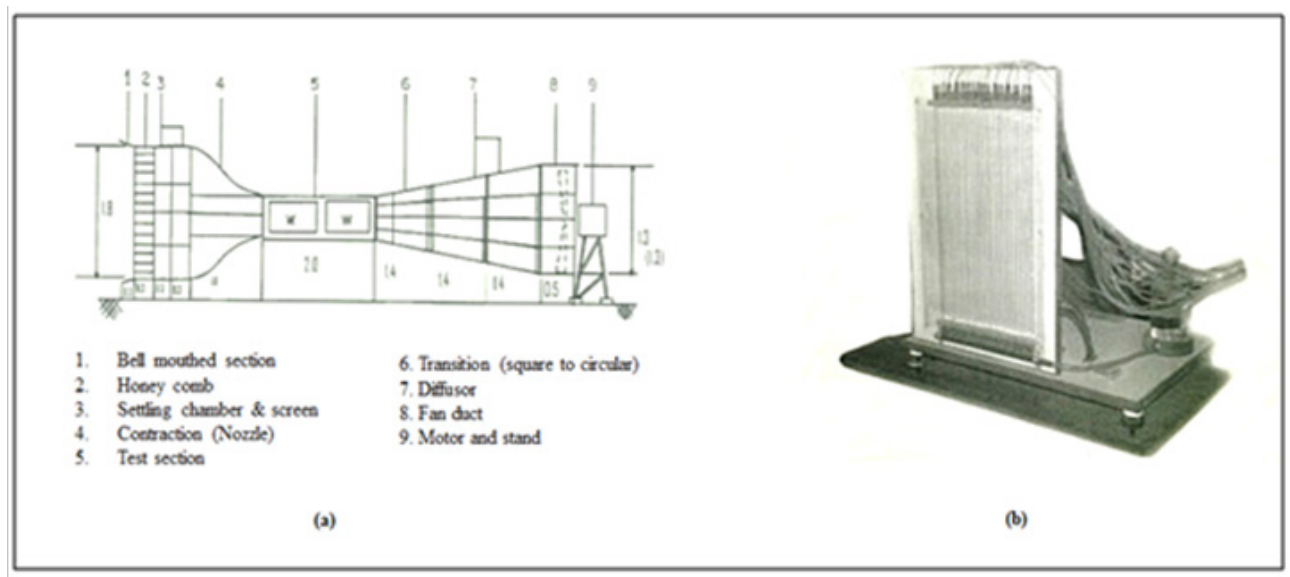

Figure 3 (A) Schematic diagram of wind tunnel, (B) Inclined tube manometer.

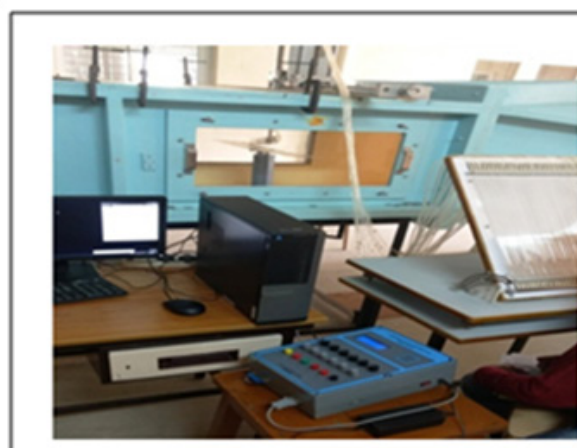

(a)

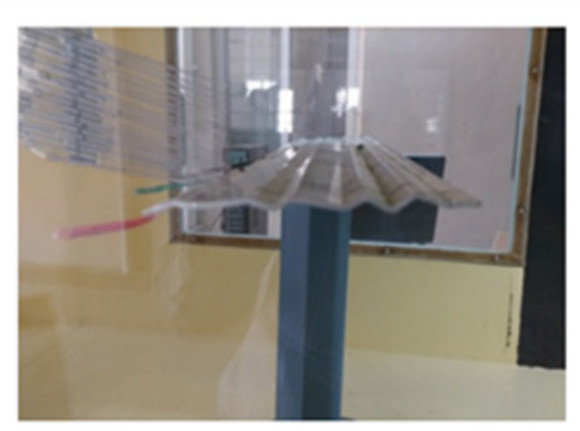

(b)

Figure 4 (A).Snapshot of experimental setup, (B) Corrugated A profile in wind tunnel.

Table 2 Wind tunnel specifications

\begin{tabular}{|c|c|c|}
\hline \multicolumn{3}{|c|}{ Nozzle and test section } \\
\hline Contraction ratio & $9: 1$ & Used to increase speed of incoming air flow \\
\hline Contraction length & $1.8 \mathrm{~m}$ & Distance over which air speed is incremented \\
\hline Stainless screens & $8 \times 16$ grid & Intended to reduce turbulence in test section \\
\hline Test section & $0.6 \mathrm{~m} \times 0.6 \mathrm{~m} \times 2 \mathrm{~m}$ & Space to install model test specimen \\
\hline Max wind speed & $50 \mathrm{~m} / \mathrm{s}$ & Design speed of wind tunnel \\
\hline \multicolumn{3}{|l|}{ Diffus or Section } \\
\hline \multicolumn{3}{|l|}{ Axial flow fan } \\
\hline Diameter & $1.3 \mathrm{~m}$ & \multirow{2}{*}{$\begin{array}{l}\text { To produce potential flow inside the tunnel at given free stream } \\
\text { condition }\end{array}$} \\
\hline RPM & 1450 & \\
\hline No of Blades & 12 & \\
\hline Fan duct diameter & $\sim 1.4 \mathrm{~m}$ & Diverging at the exit, with blade clearance, \\
\hline Motor and stand & $\sim 20 \mathrm{~kW}$ & Space to provide mounting and power axial fan \\
\hline Diffusor /Transition & & Reduces wind velocity \\
\hline \multicolumn{3}{|l|}{ Inlet section } \\
\hline Settling chamber & $1.8 \mathrm{~m} \times 1.8 \mathrm{~m}$ & Constant air flow stream ahead of contraction \\
\hline Honey comb size & $0.025 \mathrm{~m} \times 0.025 \mathrm{~m} \times 0.2 \mathrm{~m}$ & Filter particulate matter from air flow stream \\
\hline
\end{tabular}




\section{Results and discussion \\ Pressure coefficient distribution}

A comparison of the pressure coefficients for upper and lower surfaces of NACA 0010, Corrugated A and Corrugated B profiles are obtained using panel method along the chord length for $4^{0}$ angles of attack and for $\operatorname{Re} \sim 19000$. At $10 \%$ of chord length from the leading edge, the difference in pressure coefficient between upper and lower surfaces is highest, which in turn causes high lift coefficient. The pressure coefficient for NACA 0010 profile is found to decrease smoothly and for both the corrugated profiles the pressure distribution is in zig-zag pattern along the chord near the sharp edges of corrugations. Beyond $40 \%$ of chord length the pressure coefficient

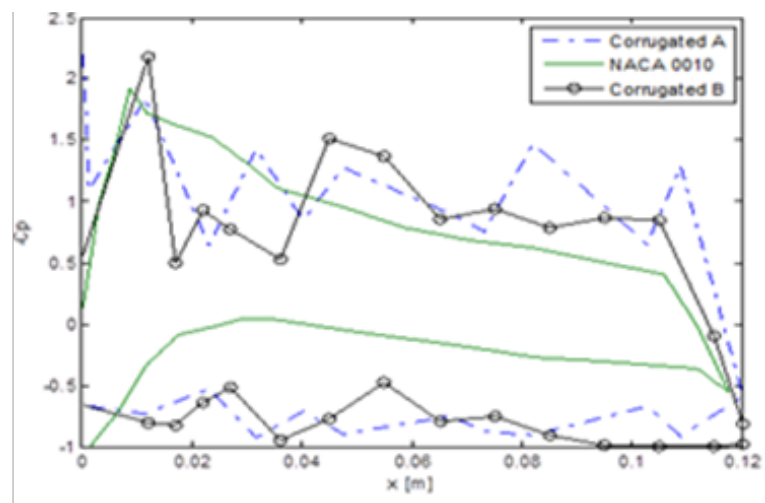

(a) rises up in Corrugated A and corrugated B profiles, in comparison to NACA 0010 profile due to the presence of corrugation in Corrugated $\mathrm{A}$ and hump in Corrugated B profiles as shown in Figure 5A. It can also be seen from Figure 5B that pressure coefficient for flat plate is smooth without any abrupt jumps along its length. For Corrugated A and Corrugated B profiles, the effect of corrugations is seen as pressure peaks due to rapid fluctuations in the velocity distribution. The contours for the pressure coefficient for three profiles are shown in Figures 6(A-C). It can be observed that pressure coefficient tends to rise with positive angle of attack however, for negative angles of attack fluctuations are observed near the corrugated surfaces. However, for NACA 0010 profile smooth contours are seen along chord length and resemble a symmetric pattern.

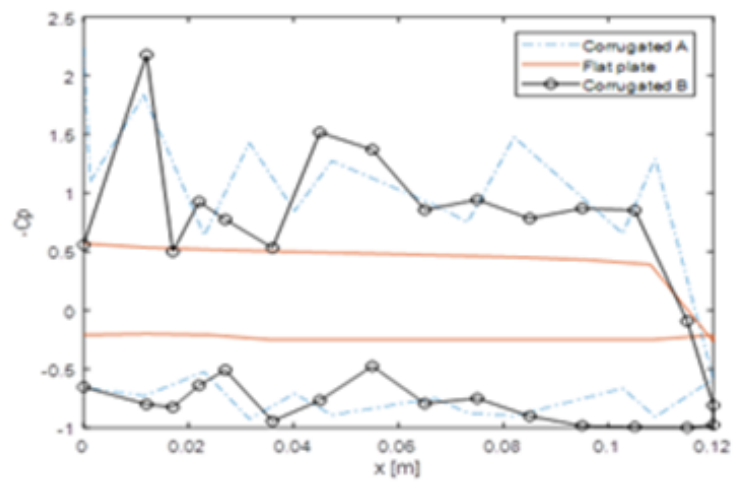

(b)

Figure 5 (A) Pressure coefficient distribution of NACA00 10, Corrugated A and Corrugated B profiles, (B) Corrugated A, Corrugated B and flat plate using 2D panel method at 40 angles of attack at $\operatorname{Re} \sim 19000$.

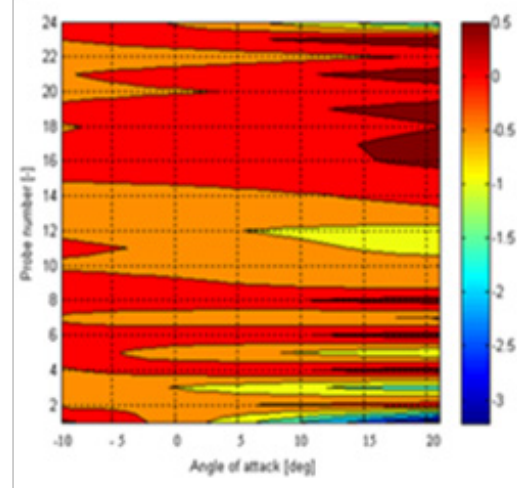

(a)

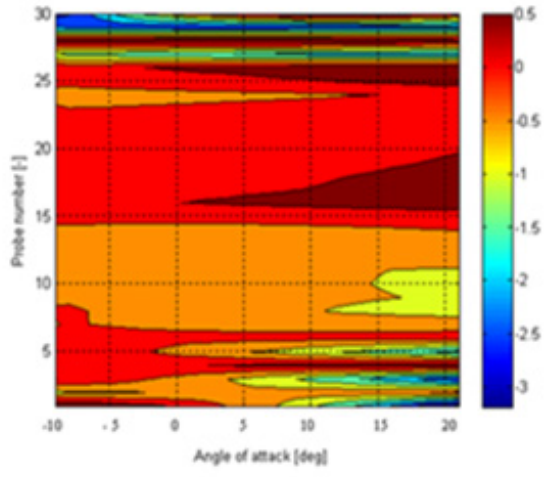

(b)

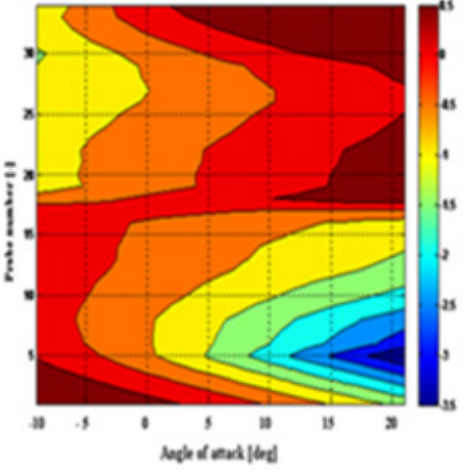

(c)

Figure 6 Pressure coefficient distribution of (A) Corrugated A (B) Corrugated B (C) NACA 0010 Probe I-14, upper surface \& probe I5-27 lower surface of corrugated A, Probe I-15, Upper surface \& probe 16-3I, Lower surface of corrugated B profile, at different angles of attack, - 100 to 200.

At $20 \%$ chord length, for both corrugated profiles the velocity increased nearly $40 \%$ for positive angle of attack. However, for negative angle of attack it is found to be less than free stream value of $20 \mathrm{~m} / \mathrm{s}$. At $40 \%$ chord length, the velocity is found to decrease in similar trend. Since NACA 0010 is a smooth and symmetric profile, there was no decrease in tangential velocity observed at $40 \%$ chord length. The pressure distribution also revealed that as the angle of attack increases the pressure coefficient is increasing on the upper surface near the rough edges of the corrugation. The trend is same for all angles of attack, from $-10^{\circ}$ to $20^{\circ}$ because of symmetry in geometry. In contrast the pressure coefficient contours for both corrugated profiles obtained using panel method on the lower surface is less prominent due to the decrease in the velocity gradient between the corrugations. Further for corrugated B profile they appear smooth but showed similar trends with corrugated $\mathrm{A}$ due to corrugations near the leading edge and broad hump near the trailing edge. The pressure distribution also revealed that as the angle of attack increases the pressure coefficient is increasing on the upper surface near the rough edges of the corrugation which is seen for all the profiles. For a given Reynolds number, the forces acting on profile surfaces are directly related to the pressure difference between the upper and lower surfaces

Further, from Figures 7A \& 7B the tangential velocity distribution on the surface of both corrugated profiles showed abrupt variations 
near the corrugations. For NACA 0010 and flat plate this trend is not seen as depicted in Figure 7C From Figures 8A \& 8B, the velocity contours for corrugated profiles the fluctuations in velocity are seen near the corrugated peaks whereas for NACA0010 profile a symmetric pattern with a continuous increase of velocity near the leading edge is observed with increasing angle of attack i.e. from $-4^{0}$ to $15^{0}$ (Figures 9(A-C) represent the contours of pressure coefficient for corrugated profiles and compared with flat plate. Similarly Figures 10(A-C), Figure 11(A-C) show the tangential velocity distribution and velocity contours of the same profiles respectively.

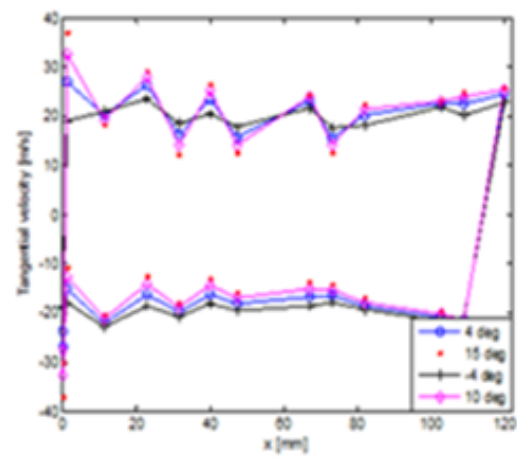

(a)

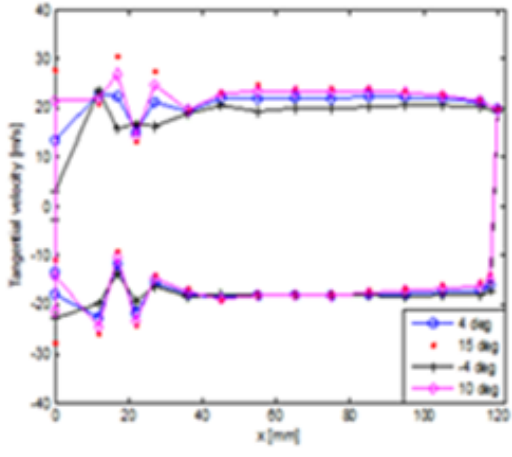

(b)

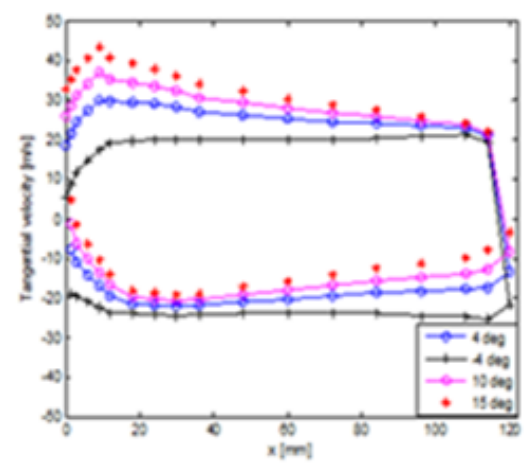

(c)

Figure 7 Tangential velocity distribution along chord (A) for Corrugated A (B) Corrugated B, (C) NACA 0010 profiles at $-4^{\circ}, 4^{\circ}, 10^{\circ}$ and $I 5^{\circ}$ angleof attack for $\operatorname{Re} \sim 1.5 \times 10^{5}$.

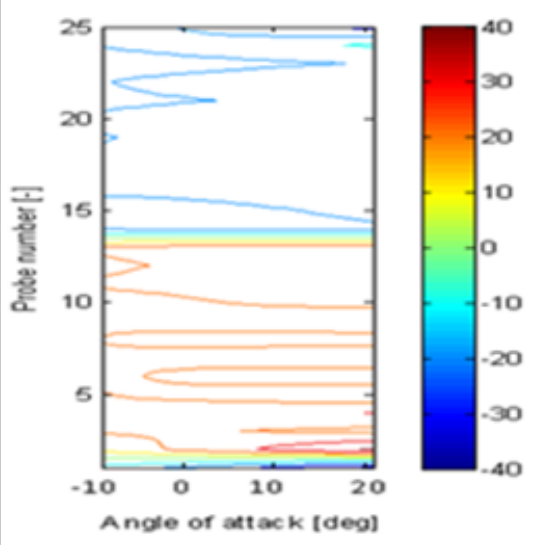

(a)

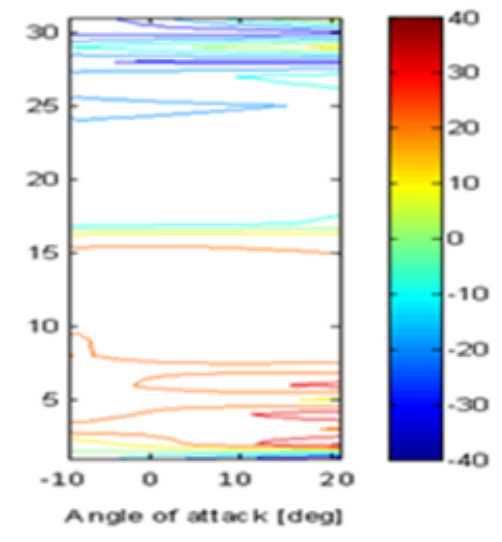

(b)

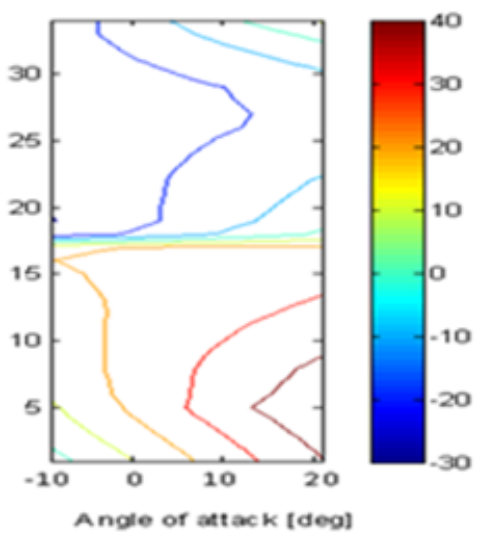

(c)

Figure 8 Velocity contours of (A) corrugated A, (B) corrugated B, (C) NACA 0010 profiles. Probe I-I4,Upper surface \& probe 15-27, Lower surface of Corrugated A, Probe I-15, Upper surface \& probe 16-31, Lower surface of Corrugated B, Probe 1-17, Upper surface \& probe 18-35 of the NACA 00I0.

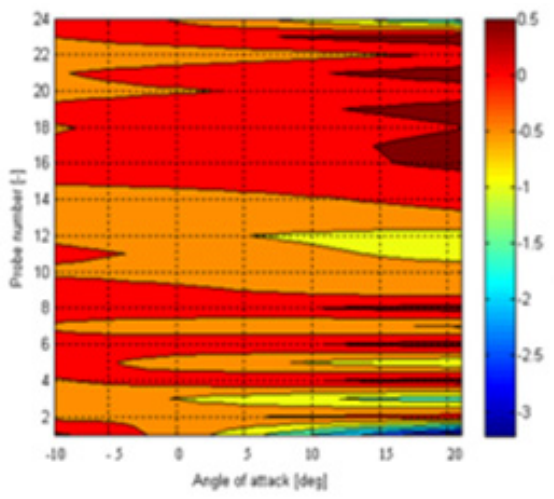

(a)

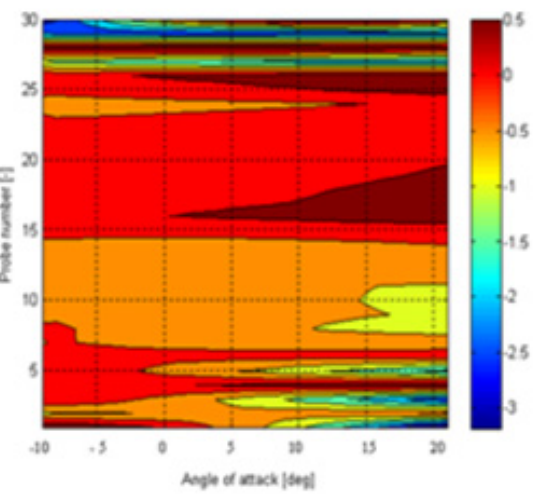

(b)

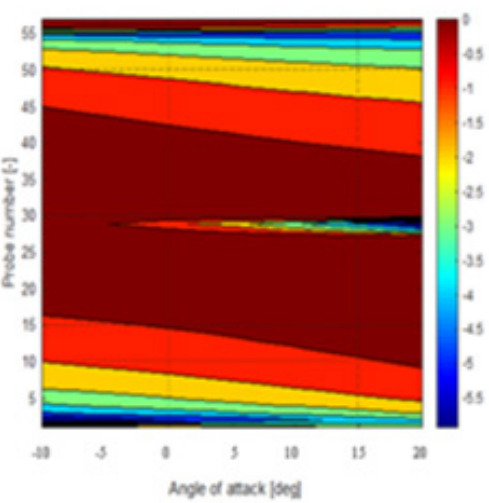

(c)

Figure 9 Pressure coefficient distribution of (A) Corrugated A (B) Corrugated B (C) Flat plate, Probe I-14, upper surface \& probe 15-27 lower surface of corrugated A, Probe I-15, Upper surface \& probe 16-31, Lower surface of corrugated B profile, at different angles of attack, - 100 to 200. 


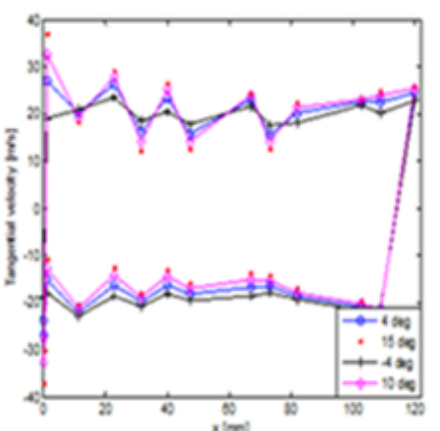

(a)

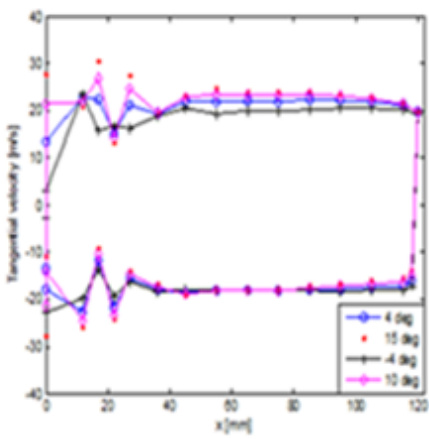

(b)

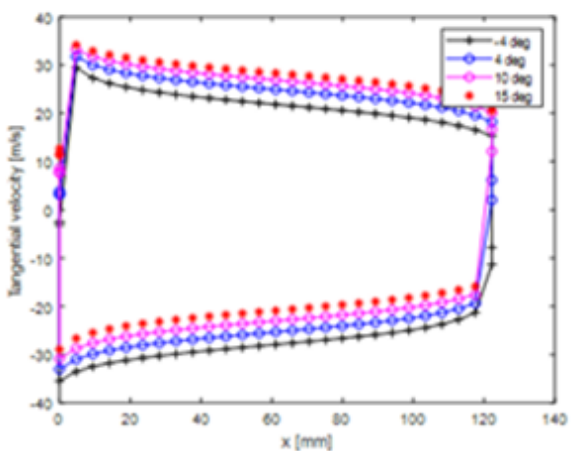

(c)

Figure 10 Tangential velocity distribution of (A) Corrugated A (B) Corrugated B (C) Flat plate, Probe I-I4, upper surface \& probe I5-27 lower surface of corrugated A, Probe I-I5, Upper surface \& probe I6-3I, Lower surface of corrugated B profile, at $-4,4$, I0 and I5 degrees angle of attack.

\section{Effect of angle of attack on aerodynamic force coefficients}

Figures 12A \& 12B shows the coefficient of lift and drag for NACA 0010 , corrugated $A$ and corrugated $B$ using panel method was evaluated between $0^{0}$ and $16^{\circ}$ angles of attack respectively. The coefficient of lift for both the corrugated profiles was found to be higher than the NACA 0010. Despite few corrugations than corrugated A profile, corrugated B profile showed higher lift for all angles of attack than the other two profiles. Until $8^{\circ}$ angles of attack, the trends for lift coefficient for all profiles are similar and found to vary almost linearly. Also, the drag coefficient for both corrugated A and corrugated B profiles were found to increase significantly than the NACA 0010 profile for angles of attack greater than $8^{0}$. It must be noted that the increase in drag coefficient for both corrugated profiles above $12^{\circ}$ angles of attack is due to the flow separation from the suction side of profiles surface at higher angles of attack. In addition the trends for drag coefficient for corrugated A and corrugated B profiles was similar up to $12^{\circ}$ angles of attack. Beyond this, rapid increase in drag coefficient of corrugated A is observed due to flow separation which is not found in corrugated $\mathrm{B}$ due to presence of hump near the trailing edge which reattaches the flow. In the Figures $13 \mathrm{a} \& \mathrm{~b}$ the experiment data for lift and drag coefficients for corrugated A and corrugated B profiles are compared with results from panel method and from (Ho \& New, 2016) The glide ratio shown in Figures 14A \&14B, Figures 15A \&15B for corrugated A, corrugated B profiles were found to be high at $5^{\circ}$ angle of attack. It is $\sim 3 \%$ more than the corrugated $\mathrm{B}$ profile but found to deviate significantly with NACA 0010 by $>10 \%$. This trend continued till $5^{0}$ angles of attack and for $\alpha>8^{0}$ the glide ratio curve resembled the NACA 0010 profile. The glide ratio for NACA 0010 found higher than both the corrugated profiles above $8^{0}$ angles of attack due to smooth surface of NACA profile which helps in flow re-attachment. Thus, glide ratio of corrugated airfoils found relatively better than the NACA 0010 profile at low positive angles of attack $\left(<8^{0}\right)$ for given Reynolds number.

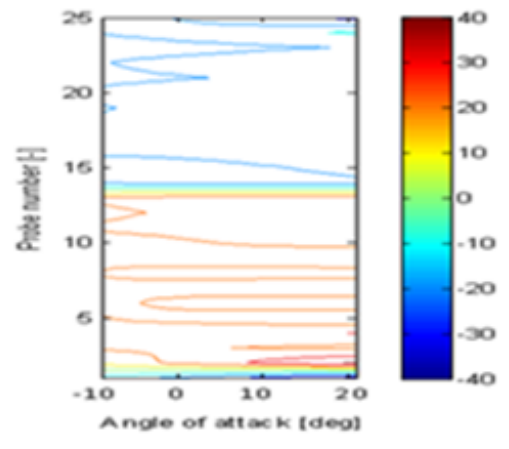

(a)

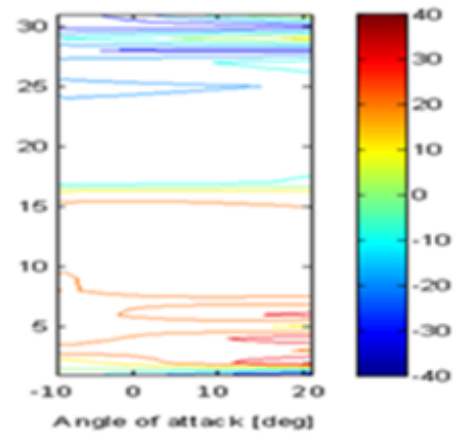

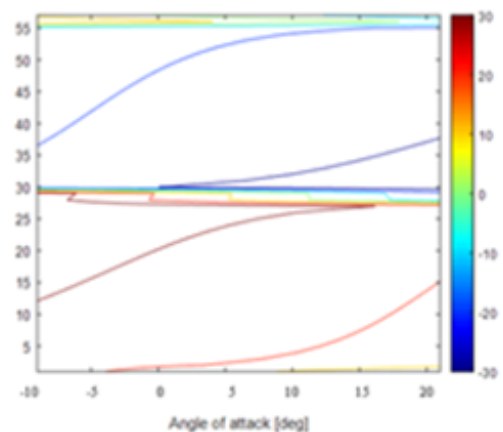

Figure I I Velocity contours of (A) corrugated A (B) corrugated B (C) Flat plate profiles. Probe I-I4,Upper surface \& probe I5-27, Lower surface of Corrugated A, Probe I-15, Upper surface \& probe 16-3I, Lower surface of Corrugated B.

\section{Validation of results}

\section{Corrugated A}

The coefficient of lift for corrugated $\mathrm{A}$ at $2^{0}$ angles of attack, was found similar by present panel method, experimental work and by Ho and New (2016). For $\alpha<10^{\circ}$ panel method results showed an increase in the lift coefficient but for $\alpha>10^{\circ}$, the results from Ho and New showed decrease in lift coefficient. It must be noted that for three profiles the drag coefficient was found nearly the same up to $\alpha<8^{0}$. However, panel method tends to predict higher drag coefficient by $\sim 30 \%$ compared to experimental work as well from Ho and New, (2016). At higher angles of attack, $\alpha>14^{0}$ all the three methods showed good agreement in the results.

\section{Corrugated B}

For this profile, the prediction of coefficient of lift by Ho and New is lower compared to present experimental and numerical work up to $\alpha \leq 12^{0}$. The present numerical results were in very close agreement with the present experimental work. Drag coefficient trends for corrugated B profile was nearly same as corrugated A profile. At angle of attack 14 degrees, Ho and New (2016) results showed, higher value for lift coefficient than the other two methods due to flow separation that was observed in his work and due to difference in the Reynolds number. 


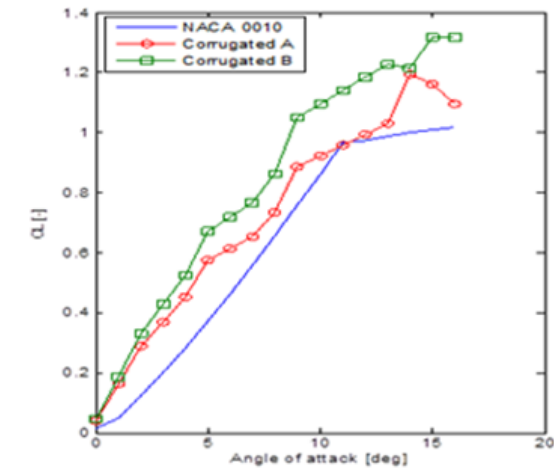

Figure 12 Results for NACA 0010 , Corrugated A and Corrugated B profiles by panel method between 00 and I60 angle of attack for Re $\sim 9000$ (A) lift (B) drag.

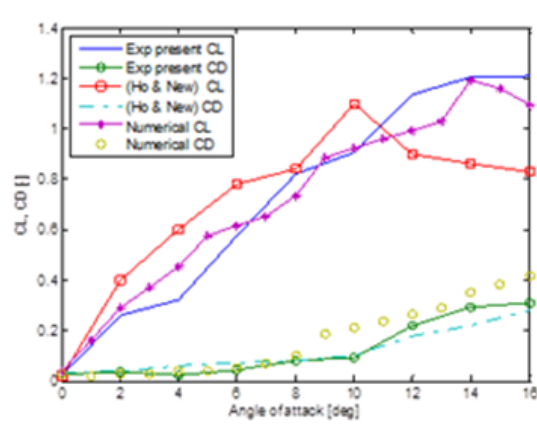

(a)

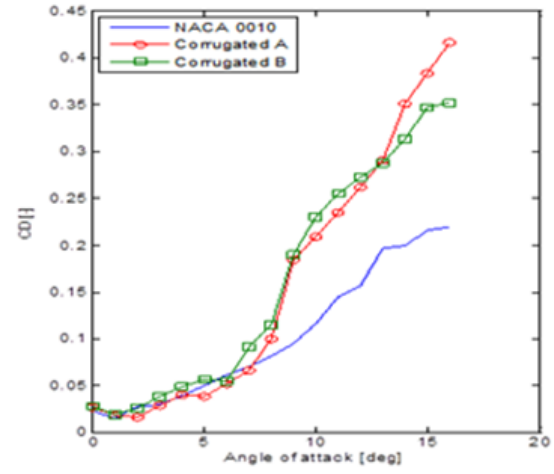

(b)

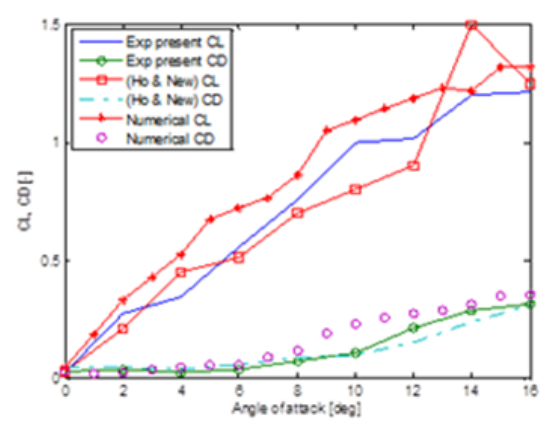

(b)

Figure 13 Comparison of lift and drag coefficient results by panel method (Re $~ 19000)$, Ho and New (2016) (Re- I4000) and present experimental work between $00-160$ angles of attack (A) Corrugated A (B) Corrugated B.

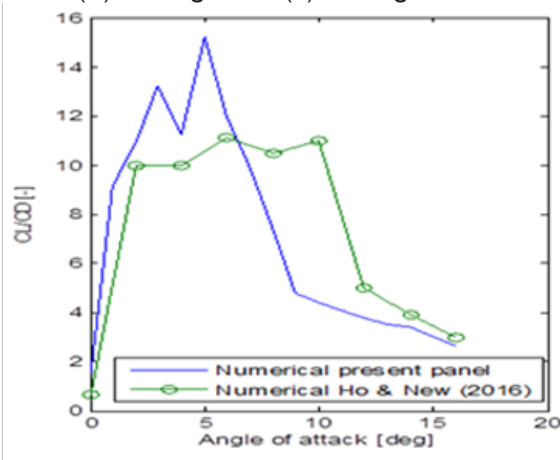

(a)

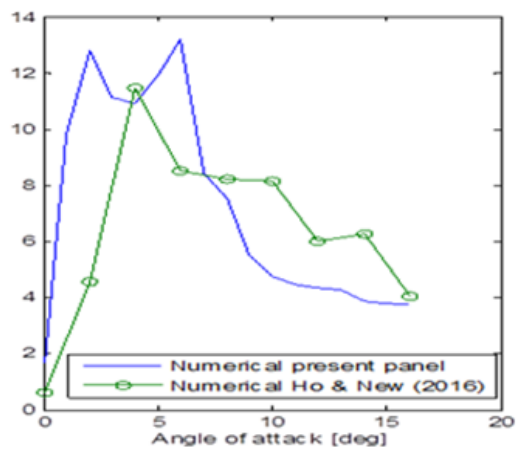

(b)

Figure 14 Comparisons of $C_{L} / C_{D}$ by present panel method at $\operatorname{Re} \sim 19000$, Ho and $\mathrm{New}(2016)$ for Re-14000, between $0^{\circ}$ and $16^{\circ}$ angles of attack. (A) Corrugated A (B) Corrugated $B$.

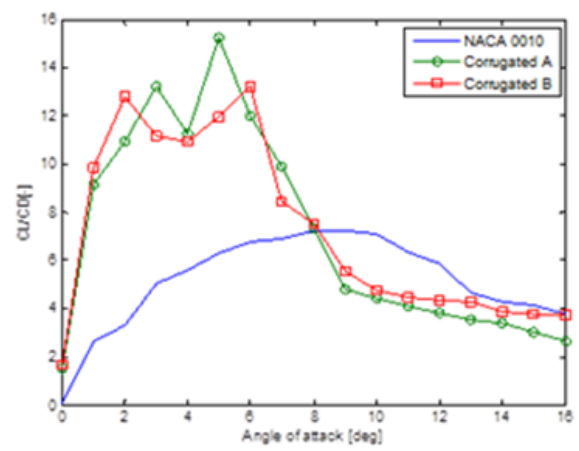

(a)

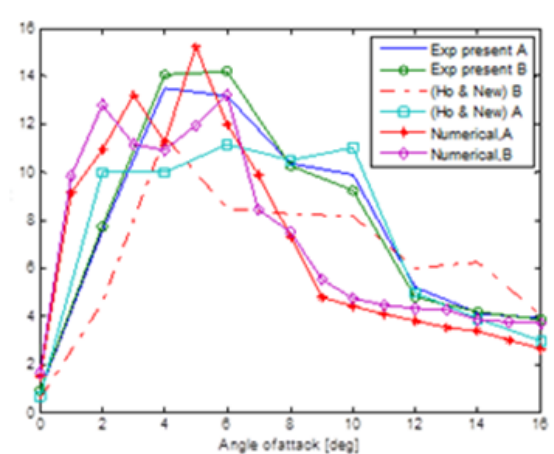

(b)

Figure 15 (A) Comparison of CL/CD for NACA 00I0, Corrugated A, and Corrugated B profiles using panel method (B) with panel method, Ho and New (2016) results and experiment data. 


\section{NACA 0010}

The results for the glide ratio $\left(\mathrm{C}_{\mathrm{L}} / \mathrm{C}_{\mathrm{D}}\right)$ of NACA 0010 profile obtained from panel method were compared with experimental work from (Murphy and $\mathrm{Hu}, 2010$ ). The Reynolds number was kept nearly same during experiment $\left(\operatorname{Re} \sim 1.5 \times 10^{5}\right.$ ) to ensure integrity in validation. The 2 -D panel method predicted $25 \%$ higher values for the coefficient of lift at higher angles of attack $\left(\alpha>10^{\circ}\right)$ and lower drag at lower angles of attack for NACA 0010. The glide ratio trends for experimental and numerical methods also show nearly the same results. The $\mathrm{C}_{\mathrm{L}}$ and $\mathrm{C}_{\mathrm{D}}$ trends for numerical and experimental results are very close $(<5 \%)$ up to $8^{0}$ angles of attack. Beyond this value the deviation in results are found to deviate high for panel method to model the flow separation phenomenon. The corrugated B profile can be used to delay stall and the flow separation for higher angles of attack as the flow reattaches with the hump. The present study also revealed that basic panel methods are able to predict aerodynamic flow phenomenon fairly accurately for $\alpha<8^{0}$. However, they are not suitable to analyze the unsteady effects and complex flow phenomenon like flow separation and vortex shedding from either leading or trailing edge of airfoil. The results also revealed that the coefficient of lift for corrugated B profile was found higher compared to remaining two profiles. The drag coefficient for both corrugated $\mathrm{A}$ and $\mathrm{B}$ profile were found to be almost same up to $12^{\circ}$ angle of attack whereas the glide ratio was found highest for corrugated A profile.

\section{Conclusion}

Basic panel method and experimental study were conducted to analyze the incompressible flow characteristics of NACA 0010 and two types of bio-inspired corrugated profiles at chord Reynolds number $1.9 \times 10^{4}$ and $1.5 \times 10^{5}$. Present work reveled that presence of corrugations on wing surface improves the flow characteristics for low to moderate Reynolds number. The reason for the increased lift coefficient at higher angles of attack for corrugated profiles is due to the large pressure difference between upper and lower surfaces which is not observed in conventional NACA profile. The panel method was able to predict the coefficient of lift and drag very well for lower angles of attack $\left(\alpha<8^{0}\right)$ and the results agreed well with computational work from Ho and New (2016). The presence of trailing edge hump for corrugated B profile increases the pressure coefficient at higher angles of attack which is similar to that observed near the trailing edge of NACA0010 profile.

\section{Acknowledgments}

None.

\section{Conflicts of interest}

The author declares no conflicts of interest.

\section{References}

1. Nawroth JC. A tissue-engineered jellyfish with bio mimetic propulsion. Nature Biotechnology. 2012;30(8):792-797.

2. Liu T, Kuykendoll K, Rhew R, et al. Avian wings. Proceeding 24th AIAA aerodynamic measurement technology and ground testing conference, Portland, OR, USA, 2004. p. 1-24.

3. Boller C, Kuo CM. Demonstration of adaptive structure performance on modular micro air vehicle. Proceedings of 51st AIAA/ASME/ASCE/ AHS/ASC structures, structural dynamics, and materials conference, Orlando, USA: FL; 2010.
4. Gao H, Hu H, Wang ZJ. Computational study of unsteady flows around dragonfly and smooth airfoils at low Reynolds numbers. Proceedings of 46th AIAA aerospace sciences meeting and exhibit. USA, 2008.

5. Gaurav S, Jain KK. Numerical investigation of fluid flow and aerodynamic performance of a dragonfly wing section for micro air vehicles (MAVs) application. Int J Innovat Scient Res. 2014;92:285292.

6. Kesel AB. Aerodynamic characteristics of dragonfly wing sections compared with technical aerofoils. J Exp Biol. 2000;203:3125-3135.

7. Kesel AB, Philippi U, Nachtigall W. Biomechanical aspects of insect wing: an analysis using the finite element method. Computers in Biology and Medicine. 1998;28(4):423-437.

8. Mingallon M, Ramaswamy S. The architecture of the dragonfly wing: A study of the structural and fluid dynamic capabilities of the Anisoptera's forewing. Proceedings of the ASME 2011 international mechanical engineering congress \& exposition: New York; 2011. p. 1-8.

9. Deubel T, Wanke S, Weber C, et al. Modelling and manufacturing of a dragonfly wing as basis for bionic research. Proceeding of 9th international design conference, Dubrovnik. 2006. p. 215-220.

10. Vargas A, Mittal R. Aerodynamic Performance of Biological Airfoils. Proceedings of 2nd Flow Control Conference, Portland, Oregon AIAA 2004. p. 2004-2319.

11. $\mathrm{Hu} \mathrm{H}$, Tamai M. Bio-inspired corrugated airfoil at low Reynolds numbers. J Aircraft. 2008;45(6):2068-2077.

12. Murphy J, Hu H. An experimental investigation on a bio-inspired corrugated airfoil. Proceedings of 47th AIAA aerospace sciences meeting and exhibit, Orlando, FL, USA, 2009.

13. Levy DE, Seifert A. Simplified dragonfly airfoil aerodynamics at Reynolds numbers below 8000. Phys Fluids 2009;21(7): 10.1063.

14. Levy DE, Seifert A. Parameter study of simplified dragonfly airfoil geometry at Reynolds number of 6000. J Theor Biol. 2010;266(4):691702 .

15. Shi SX. An experimental study of flow around a bio-inspired airfoil at Reynolds number 2.0x103. J Hydrodynamics. 2010;243:410-419.

16. Webb C, Dong H, Mittal R. Motion kinematics effects on aerodynamic performance of bio-inspired wing sections in ultra low Reynolds number flow. Proceedings of 47th AIAA aerospace sciences meeting including the new horizons forum and aerospace exhibition. Orlando, FL, USA, 2009.

17. Hord K, Lian YS. Numerical investigation of the aerodynamic and structural characteristics of a corrugated airfoil. Journal of Aircraft 2010;49(3):749-757.

18. Kwok M, Mittal R. Experimental investigation of the aerodynamics of a modelled dragonfly wing section. Proceedings of AIAA Region-I MA Students Conference. 2005.

19. Okamoto M, Yasuda K, Azuma A. Aerodynamic characteristics of the wings and body of a dragonfly. J Exp Biology. 1996;199:281-294.

20. Rees CJC. Form and function in corrugated insect wings. Nature 1975a;256:200-203.

21. Rees CJC. Aerodynamic properties of an insect wing section and a smooth aerofoil compared. Nature. 1975b;258:141-142.

22. Wu JH, Sun M. Unsteady aerodynamic forces of a flapping wing. $J$ Exp Biology. 2004;207:1137-1150.

23. Chen YH, Skote M. Gliding performance of 3-D corrugated dragonfly wing with spanwise variation. Journal of Fluid and Structures. 2015;62:1-13. 
24. Ho WH, New TH. Unsteady numerical investigation of two different corrugated airfoils. Proceedings of IMechE Part G: J Aerospace Engineering. 2016;0(0):1-15.

25. New TH. Effects of corrugated aerofoil surface features on flowseparation control. AIAA Journal. 2014;521:206-211.

26. Houghton EL, Carpenter PW. Aerodynamics for engineering students. 6th Ed. Butterworth-Heinemann Publication, Oxford, UK, 2013.

27. Anderson JD. Fundamentals of Aerodynamics. 5th edn. New York: McGraw Hills Inc; 2012.
28. Muralidhar K, Sundararajan TT. Computational Fluid Flow and Heat Transfer. 2nd Ed. Narosa Publishing House Ltd. New Delhi, India, 2011.

29. Murphy J, Hu H. An experimental study of a bio inspired corrugated airfoil for micro air vehicle applications. Exp Fluids. 2010;49(2):531546.

30. Pamadi BN, Performance, Stability, Dynamics, and Control of Airplanes. Second Edition. AIAA. Inc Reston VA. 2004. p. 20191-4344. 